Paul Alivisatos

University of California, Berkeley, USA

Inorganic nanocrystals with well defined shapes are important for understanding basic size-dependent scaling laws, and may be useful in a wide range of application. Methods for controlling the shapes of inorganic nanocrystals are evolving rapidly. This talk will focus on a strategy that involves pyrolysis of organometallic precurso in mixtures of hot organic surfactants. The surfactant mixtures can be used to control the growth rates of differnet facets of the nanocrystals, allowing for wide tunability of shape. This will be illustrated with CdSe and Co nanocrystals. Both of these materials show pronounced variation of fundamental properties with aspect ratio. The nanorods can be aligned in a variety of ways. For instance, monolayers of

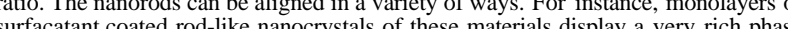
作 diagram, anase copolymers can be used orient the rods. rods at the tetrahedral angle, will always spontaneously align perpendicular to surface. The possible application of these aligend nanorods in
photovoltaics, and light emitting diodes will be described briefly.

\section{Molecular-Level Devices and Machines}

Vincenzo Balzani

Università di Bologna, Italy

A molecular-level device can be defined as an assembly of a discrete number of molecular components (that is, a supramolecular structure) designed to achieve a specific function. Each molecular component performs a single act, while the entire supramolecular structure performs a more complex function, which results from the cooperation of the various molecular components.

Molecular-level devices operate via electronic and/or nuclear rearrangements, and like macroscopic devices they need energy to operate and signals to communicate with the operator. Light is the most important answer to this dual requirement, as shown by Nature where photons are used as energy by the devices responsible for photosynthetic processes and as signals by the devices responsible for vision-related processes. Another useful technique to cause and to monitor the occurrence of electronic and nuclear rearrangements in molecular and supramolecular systems is electrochemistry.

In the last few years prototypes of very simple molecular-level machines and molecular-level components related to the construction of molecular-based (chemical) computers have been developed by several research groups. ${ }^{1}$ Examples of molecular-level devices and machines investigated in our laboratory will be illustrated and discussed.

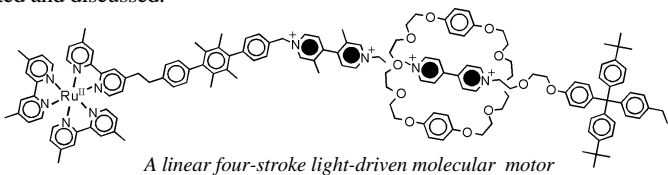

1(a) Balzani, V. "Molecular Machines", McGraw-Hill 2000 Yearbook of Science and Technology, 1999, 259-262 (a) Balzani, V. "Molecular Machines", McGraw-Hill 2000 Yearbook of Science and Technology, 1999, $259-2$ Ballardini, R.; Balzani, V.; Credi, A.; Gandolfi, M.T.; Venturi M.; Acc. Chem. Res., in press.

\section{$\underset{\text { acids }}{\text { Nano }}$}

Frank F. Bier, Ralph Hölzel, Markus von Nickisch-Rosenegk, Christian Heise, Eva Ehrentreich-Förster, Nenad Gajovic-Eichelmann, Xenia Marschan

Fraunhofer-Institut für Biomedizinische Technik, AMBT, Abt. Molekulare Bioanalytik und Bioelektronik, Bergholz-Rehbrücke, Germany

For many applications manufactured surfaces with engineered structures in the nano-meter range are desirable. The properties of nucleic acids makes them perfect material for this purpose: Nucleic acids have a regular structure with a 0.34 $\mathrm{nm}$ period, through its sequence it is addressable in a nanometer range.

Technically we propose to immobilise DNA-oligomers on multiple points in an ordered way. It is important to retain its functionality, the ability to hybridise to complementary nucleic acid or to get recognised by binding proteins. Longer DNA fragments are stretched and anchored by hybridisation between a given structure of oligonucleotides in the $\mu \mathrm{m}$ range. Here we present and compare different approaches to realise such oligonucleotide
structures. Employed techniques include micropainting, photochemistry, selforganised monolayers on metal surfaces and others.

Scanning probe microscopy (AFM and SNOM) and laser scanning microscopy (LSM) will be used to analyse these structures. First results are reported.

Beside possible applications of the DNA-modified surfaces as DNA arrays with ingene resolution, it will provide a framework for building larger rational assemblies used e.g. in new biosensors, coupled enzyme reactions or even DNA based computing and macromolecular machines.

\section{Atomic Resolution Imaging of Nucleic Acid /Taxol@ Complexes}

\section{Gerlinde Bischof}

Martin Luther University Halle-Wittenberg, Institute of Biochemistry

The nucleic acid activity of Taxol® was investigated with synthetic and natural oligo- and polynucleotides. The examinations were performed with spectroscopic and calorimetric methods as well as atomic force microscopy (AFM) investigations. In the study, Taxol ${ }^{\circledR}$ shows molecular recognition of AT nucleotides with a high affinity to homologous (dA)_(dT) sequences, while no interaction with GC nucleotides could be observed.

High-resolution imaging of the DNA/drug complexes were investigated by AFM using Digital Instrument Nanoscope III with a resolution of up to $30 \times 30 \mathrm{~nm}$ (see Fig. 1). The monolayers of the samples were typically investigated with given conditions: constant force contact or tapping mode; standard $\mathrm{Si}_{3} \mathrm{~N}_{4}$ - or Si-cantilever; graphite, mica or glass substrate; in air; room temperature.

AFM observation of the complexes shows the formation of strands and toroidal structures (approximately 200-300 nm diameter). By investigating pure nucleic acid samples without drug, displaying only strands, and pure Taxol with nucleic samples without drug, displaying only strands, and pure Taxolß without nucleic acids, displaying agglomeration of the molecules up to $20 \mathrm{~nm}$ diameter, no Regular arrangements of DNA molecules are already visualized by AFM (or other nanoscopic techniques). Due to a certain degree of order preserved during evaporation, they often form parallel strands on the substrate.

We, as well as others, observed this in previous studies, but we observed also toroidal formations under different conditions (e.g., nucleic acids in presence of drugs, proteins, others). It seems to be characteristic of long and soft molecules, resulting from anisotropic conditions, to form supracoiled structures turning into spherical shapes.

By zooming in the strand-like region of the topics, thick strands aligned to more slim strands are visible. Partly association of drug molecules to the strands could be slim strands are visible. Partly association of drug molecules to the strands could be an interpretation to the different thicknesses of the strands. Based on the physicochenical to the different thicknesses of the strands. Based on the performed assisted by molecular modeling. They are in favor of rather specific Taxol

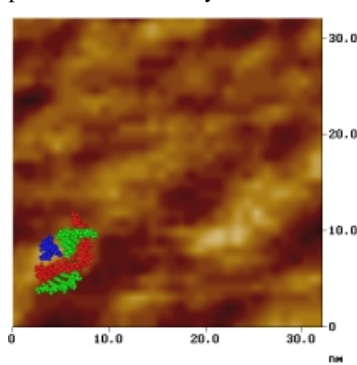
major groove interactions.

Fig. 1: High-resolution imaging of the poly $(d A)$ poly $(d T) /$ Taxol@ complex with molecular 


\section{DNA-based constructions on gold surfaces}

\section{A. Csáki, G. Maubach, R. Möller, J.M.Köhler, W. Fritzsche}

Molecular Nanotechnology Group, IPHT Jena, Germany

The implementation of biopolymers, such as nucleic acids, provides an opportunity for nanotechnology. Synthetic architectures of DNA-molecules, novel methods for labeling with DNA-metalic nanoparticles and immobilization-techniques are the basic principles of our approach. This basic essentials are combined in molecular building blocks for various applications in nanobiotechnology.

Adressable parts of molecular construction are modified synthetic oligonucleotides. For immobilization, they bound to the metall surfaces as SAMs. Long nucleic acids act as frame for specific constructions. This molecules feature molecular-biologica processability, combinatorial information capacity and the ability for selforganisation. Important elements of the construction blocks are DNA-modified metal nanoparticles. They act as sequence-specific markers with complementary ites. In this sites. immobilization and the successive elongation of DNA-chains, and the manipulatin of long DNA. Scanning force microscopy was used as standard method for the

The combination of DNA-based molecular construction and top-down techniques in a microsystems provide a large potential for novel labeling techniques in molecular diagnostics, for construction-technology towards future electronic devices or for single molecule investigations and manipulations.
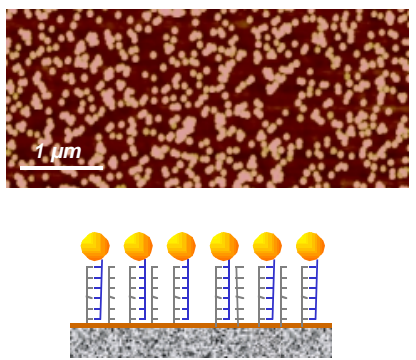

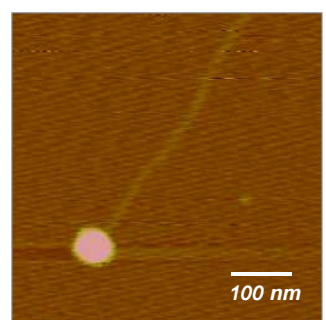

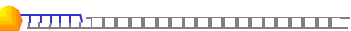

\section{Nanoparticle-based optical read-out for DNA-chip technology}

\section{W. Fritzsche, A. Csaki, R. Möller, J. Reichert, J.M. Köhler}

Molecular Nanotechnology Group, IPHT Jena, Germany

DNA-chips and -microarrays are promising tools in DNA diagnostics. Using high parallelization, detection of DNA-sequences is possible with significantly higher speed and at greatly decreased costs. The chip-based screening for specific biomolecular binding applies usually a fluorescence signal. Fluorescence labeling is limited regarding the dye stability and the environment-dependent signal (which hampers quantification), and there is the need for a sophisticated optical equipment
for detecting the signals. We think that the fluorescence label could be complemented by colloidal gold particles. Such nanometer-sized gold particles are well established as labels in electron and light microscopy. Techniques for well established as labels in electron and light microscopy. Techniques for mmobilization of proteins or DNA on the surface of the particles are available. The next step towards a biochip based on gold labeling is the use of microstructured
substrates, which opens the way for high-density arrays. substrates, which opens the way for high-density arrays.

We demonstrate the optical detection of nanoparticle-labeled DNA on microstructured chip substrates. The signal can be imaged using conventional brightfield microscopy, and on a much smaller time scale (ms) compared to fluorescene chips. The use of metal enhancement increases the optical signal, which will help to extend the dynamic range toward very low particle densities. Applying this enhancement, single particles could be visualized in conventional optical microscopes.
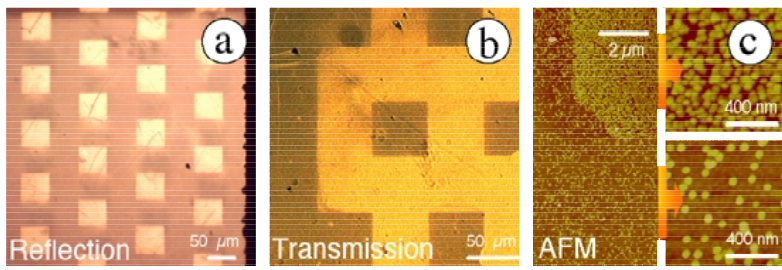

Microstructured DNA-arrays on glass after incubation with complementar gold-labeled 1

Reichert, A. Csaki, R. Möller, J.M. Köhler, W. Fritzsche: Analytical Chemistry 72 (24) 2000, 6025-6029.
Ultrafast opto-optical logic elements on a nano-scale basis

\section{K.-H. Feller, E. Gaizauskas, V. Malyshev}

University of Applied Sciences Jena, Germany

The research into appropriate new organic materials for application in photonics is one of the main subject of our work. We concentrated our investigations in this connection to such high molecular organic systems like J-aggregates. Our investigations have shown that $\mathrm{J}$ - aggregates are very promising candidates for their application in photonics. Their main advantage is the combination of huge nonlinear optical susceptibilities $\left(x^{(3)} \sim 10^{-8} \mathrm{esu}\right.$, which is of the order of magnitude of the best anorganic NLO materials) and extreme short response time (sub-ps to fs- region). Via the interaction of such competitive processes like saturation of absorption and exciton-exciton-annihilation the mechanism of the decisive nonlinear optical processes is intensity dependent itself. Examplary for this is the intensity dependent ultrafast non-exponential relaxation kinetics (see Figure 1) and the anormal wavelength dependence of the excitation intensity in the case of degenerate foumixing (DFWM) (the power index of the converted signal decreases in dependence of the excitation intensity from 3 out of resonance to approximately 1 in resonance) and an intensity dependent spectral shift of the DFWM signals. This enables short switching times for signal processing at moderate intensities by means of the huge non-linear effectivity whereas the intensity acts a new parameter of freedom.

The explanation of the processes is given by means of non-perturbational modell, which includes exciton-exciton-annihilation, two-photon-

resonance processes, electron-phonon-coupling as well as coherent nonMarkovian effects in the ultra-short time region.

Recently we have shown, that in J-aggregates feedback-free bistabilities are possible too, based on which switches in transmission regime could be built up. In this case we see a hysteresis-like dependence of the input and output intensity enabling the
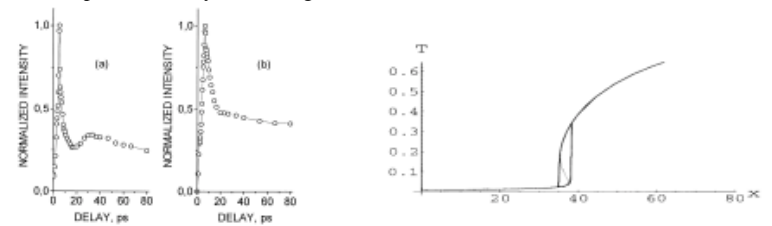
Figure 1: Non-exponential relaxation kinetics of the
signals of degenerate four-wave-mixing in PIC-J signals of degenerate four-wave-mixing in PIC-
aggregates at different irradiation intensities: corresponds to the rate of $1: 10$ of excited molecules Figure 2: Hysterese of the optical transmittivity T of an ultrathin layer of $\mathrm{J}$ - aggregates in dependence on the irr
solution)

\section{Structure of DNA/Dendrimer Complexes}

I. Gössl*, L. Shu ${ }^{+}$, A. D. Schlüter ${ }^{+}$and J. P. Rabe*

* Humboldt University Berlin, Germany

+ Free University Berlin, Germany

The condensation and aggregation of DNA molecules into globular structures by different multivalent ions, polyamines, cationic lipids and cationic polymers are of fundamental interest in understanding the packing mechanisms of DNA molecules in vivo ${ }^{1}$. Furthermore most of these synthetic molecules are developed for DNA vectors in gene therapy. One efficient synthetic gene-delivery agent is the
Poly(amidoamine) dendrimer. Size and morphology of these self-assembled nonviral gene delivery systems (DNA/dendrimer) are not fully understood, although size and morphology may influence the efficiency of transfection ${ }^{3}$.

In the present study we used cylindrically shaped dendrimers and analyzed their interaction with single double stranded DNA molecules. Attached to a polystyrene interaction with single double stranded DNA molecules. Attached to a polystyrene backbone are dendritic substituents forming the cylindrically shaped dendrimer. A
high positive charge density of these dendrimers is achieved by the protonized high positive charge density of these dendrimers is achieved by the protonized
amine groups on the periphery. As a function of dendron generation 2,3 and 4 the
charge densities, radii and persistence lengths vary. Besides these molecular charge densities, radii and persistence lengths vary. Besides these molecular properties also environmental parameters like $\mathrm{pH}$ and substrate were studied regarding their influence on the structure of the self-assembled complexes of DNA
molecules and dendrimers. The formed complexes were adsorbed on bare mica and functionalized mica surfaces, where they were analyzed using scanning force microscope in air.

A linear dependence between the used length of the DNA molecule for complex formation and the contour length of the complex is observed. Evidence for the structure of the complexes can be obtained analyzing this linear dependence as well as height measurements and contour length distributions of DNA molecules, dendrimers and complexes. The experiments discussed here show, that for certain DNA/dendrimer concentrations DNA is wrapping around the dendrimer of DNA/dendrimer concentrations DNA is wrapping
generation 2, 3 and 4 forming a well structured complex. 
Handling of Femtodroplets and Single DNA Molecules for Studies on Single Molecule Enzyme Reactions

B. Nasanshargal, P. Sharma, B. Schäfer, K. O. Greulich

Institute for Molecular Biotechnology Jena, Germany

Nanomanipulation of droplets of enzyme- and substrate solutions, in combination with fluorescence microscopy, allows to study reactions catalyzed by a few enzyme molecules. One example is the conversion of nonfluorescing $\mathrm{NAD}^{+}$in fluorescing NADH by a few molecules of lactate dehydrogenase in femtodroplets. Since effects of nearby surfaces often make quantitative observation difficult, three different techniques, all involving femtodroplet handling, are compared. At least by one of them, Lineweaver Burk plots are obtained for single enzyme molecules yielding approximately the macroscopic kinetic constants of the enzyme. Direct nanohandling is required when reactions on single DNA molecules are observed. Here, an individual, fluorescently labeled DNA molecule is coupled to a microbead (approx. 1 micrometer in diameter) which can be held and moved along complex trajectories by optical tweezers. Using a hydrodynamic flow or an electrostatic field, the DNA molecule, which otherwise collapses into a globular structure, can be stretched into an elongated filament. The bead itself is non fluorescing, but becomes visible when DNA is wound around it. Stretched DNA molecules can be cut into sequence specific patterns by restriction endonucleases such as Apa1, Sma1, EcoR1, Mle 1 and Mfu 1.The figure shows three of these restriction reactions. Individual DNA molecules with different sequences can be distinguished from each other.
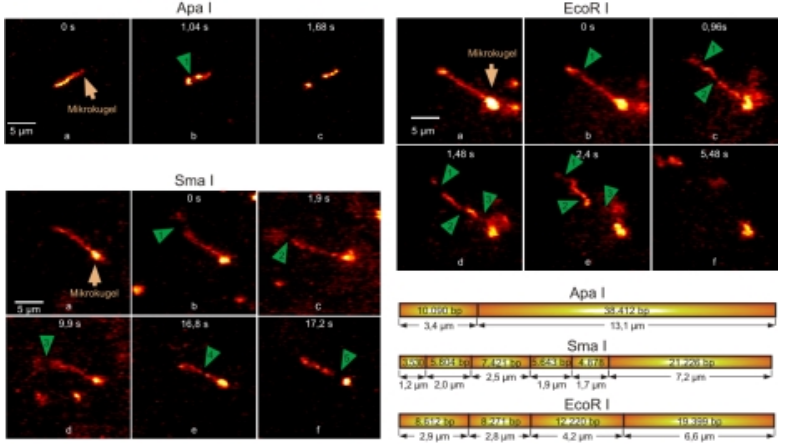

Nanoscale Biomolecular Arrays

\section{Eric Henderson}

Iowa State University / BioForce Laboratory, Ames (IA), USA

Ultraminiaturization of biomolecular arrays affords several key advantages. For example, increases in throughput can be realized concurrently with decreases in production and utilization costs. However there are numerous consideration involved in the construction and implementation of nanoscale biomolecular arrays. Our group has developed tools to facilitate the construction of protein and nucleic acid arrays with micron and sub-micron spot size. As the arrays become smaller, the limits of resolution of existing methods for detection (e.g far field optics) prevent their use and novel detection methods (e . a., atomic force microscopy: AFM) prevent their use nanoscale biomolecular arrays will be discussed and projections for their acceptance and utilization will be presented.

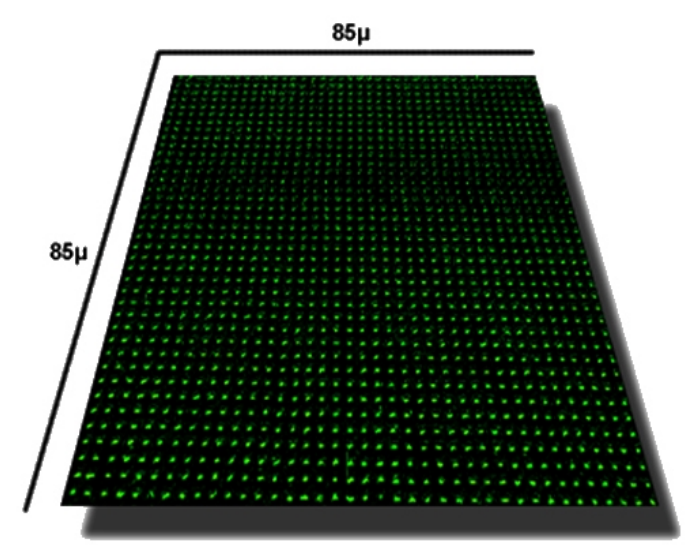

1521 Spot NanoArray
Detection of Single Molecules by Gold-Silver and Gold-Gold Staining Methods

Gerhard W. Hacker

Landesklinken Salzburg, Salzburg, Austria

Two decades ago, Gorm Danscher (Aarhus, Denmark) introduced silver enhancement (autometallography, AMG) to detect catalytic tissue metals in microscopy, as a prerequisite for obtaining a higher sensitivity in immunogold applications. Combining this reaction with colloidal gold-labeled enzyme histochemistry and immunohistochemistry (IHC) was published simultaneously in 1983 by Danscher and Clive Holgate et al. (UK), respectively, and are considered real breakthroughs which fifteen years later lead first proofs of molecular detection sensitivity in the DNA detection field. The original IHC technique had been named immunogold-silver staining (IGSS) and, already at that time, did dramatically improve sensitivity and detection efreciency. Under the LM, conglomerations of gold particles enbedded in silver appeaction products of most enzyme-labeled preparations, thus offering a variety of advantages in . improve the gold-silver technology for various kinds of applications. Starting with IHC, in proction of gold crystals sure Inc:- ht /// goves, In.; catary the dive techniques of mocular morpholosy. Application of tyramide sorld of amplification (TSA ${ }^{\oplus}$; Perkin Elmer Life Sciences, http://lifesciences perkinelmercom), and of gold ion-based AMG to smoothly increase the size of the clustered gold label, have of gold ion-based AMG to smoothly increase the size of the clustered gold label, have recently expanded the scope of applications to hitherto unknown, single molecule
sensitivity at the level of DNA detection by ISH without the need for prior PCR. Most recently applications in molecular biology (Western blotting) and microchip array recently applications in molecular biology (Western blotting) and microchip array greater ease, higher sensitivity and good reproducibility.

Single copies of human papillomavirus HPV 16/16 TSA-amplified gol Published by Graf A.-H., Hacker G.W. et al. in Applied Immunohistochemistry and Molecular
Morphology $800-309$ 2000 Copyright by Morphology 8, 300-309, 2000. Copyright by

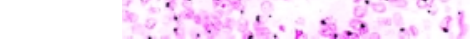

Acknowledgement: I would like to express my deep thankfulness to my collaborators on IGSS methodology, Tubbs (OH, USA), James Hainfeld (NY, USA), Annie, DM. Cherng (HK), Ingerge Zehbe (D), Huici SU (CA, USA), Cornelia Hauser-Kronberger, Otto Dietze, Anton-H. Graf, Günter Schwamberger (Salzburg, A)
DNA-directed assembly of metal particles for biosensing and nanomaterials synthesis

\section{D. Keating}

Pennsylvania State University, USA

This presentation will focus on the use of deoxyribonucleic acid (DNA) to direct the assembly of metal particles. DNA has been attached to the surfaces of colloidal Au spheres as well as metal nanowires several microns in length. The ability of particlebound DNA to selectively and reversibly bind to complementary sequences has been demonstrated. When complementary sequences are located on two different metal surfaces, hybridization can be used to direct the assembly of particles in metalion or onto macroscopic surfaces. This directed assembly can be exploited either for bioanalysis or for materials synthesis. For example, the assembly process either for bioanalysis or for materials synthesis. For example, the assembly process can report on the presence of a DNA sequence of interest, with particles acting as
amplification tags to facilitate detection. We have used this approach to increase the sensitivity of surface plasmon resonance (SPR). When the assembly of 12-nm Au particles is coupled to binding of an analyte DNA sequence, we observe a thousandfold improvement in sensitivity as compared to the unamplified SPR assay. Nanoparticle-amplified SPR can be done in an imaging format, and may have application to DNA microarrays.

A second application of DNA-directed assembly is in the deterministic construction of nanostructured materials. We are particularly interested in the potential of DNA hybridization to selectively connect metal nanowires into functional electronic devices. To this end, we are investigating DNA assembly and hybridization on nanowires, and the construction of very simple DNA-linked structures. It is possible to selectively derivatize $\mathrm{Au}$ and $\mathrm{Pt}$ surfaces; this chemistry has been used to immobilize DNA on only the central segments of Pt-Au-Pt striped nanowires. imple chemistry; optimization of this process will be discussed. 


\section{Molecular Nanotechnology beyond Genes and Beads}

\section{J. M. Köhler}

Technical University Ilmenau

The term Nanotechnology is related now to two rather different fields of technical sciences: the nanofabrication dealing with objects in the length of nanometers, and the nanolaboratory techniques in chemistry and in molecular biology related to the miniaturization of liquid volumes down to the nl-range.

DNA is of interest in both fields. Nucleic acids are fascinating objects with a lot of properties making them suited for the formation of complex molecular constructions. Therefore, the molecular basis of genes is an important model for molecular nanotechnology, on one hand. On the other hand, DNA is the most popular target in the application of nanoliter technology in labs. The development of biochips and of new classes of miniaturized lab devices for the technical read-out of biomolecular information is strongly related to DNA technology.

Nanobeads - particles with diameters below $100 \mathrm{~nm}$ - are the typical objects of mesoscopic physics. They are standing in their properties between molecules an extended solids, between classical and quantum world. So, they are a symbol for the ultimate down-scaling of solid state technology. In addition, they are recently used as labels for biomolecular recognition and they are shown to be suited for molecular construction.

In the paper, the use of nanoparticles as complementary elements to macromolecules is discussed with the background of the application of them at macromolecules is discussed with the background of the application of them planar solid suffaces. It is shown, that nanoparticles are in a bridge function between single molecule techmiques particles are of particular and their physical solid state properties like mechanical stability, electrical conductibility, and their interaction behaviour with photons. It will be suggested, that the stiffness and the mesoscalic properties of nanoparticles will become key parameters for molecular construction and for the development of artificial functional nano systems, in particular, in connection with the use of solid state technologies based on planar technology.

\section{DNA-nanoparticle assemblies: structural basis for the optical and electrical properties}

\section{Anne A. Lazarides and Chad A. Mirkin}

Northwestern University, USA

A major goal of the analytical DNA detection community is to develop a highly selective method of detecting genomic DNA without the aid of target amplification schemes such as PCR. Assays that use DNA-functionalized nanoparticle probes display single-base mismatch selectivity (1), and offer promise of providing ultrahigh sensitivity on the basis of the unique optical, catalytic, and electrical properties of metal particles and their assemblies. Success will depend upon understanding how optical and electrical properties depend upon the structure of nanoparticle assemblies and how DNA can direct the assembly of these structures.

Candidate assemblies include nanoparticles assembled on chips and nanoparticle aggregates assembled in colloidal suspensions. Models of the extinctive, scattering, and electrical transport properties of these assemblies have been developed (2). In and electrical transport properties of these assemblies have been developed (2). In both dry and aqueous assemblies, interparticle separations have been measured using small angle X-ray scattering (3). DNA has been observed to control
interparticle separations in both cases, with rigid duplexes in the aqueous phase and interparticle separations in both cases, with rigid duplexes in the aqueous phase and
a disordered organic spacer layer in the dry assemblies. When the structural data is a disordered organic spacer layer in the dry assemblies. When the structural data is
incorporated into the electrodynamic and charge transport models, observed optical incorporated into the electrodynamic and charge transport
spectra and charging energies are successfully reproduced.

1. J.J. Storhoff, R. Elghanian, R. C. Mucic, C. A. Mirkin, R. L. Letsinger, J. Am. Chem. Soc. 120, 1959 (1998); T. A. Taton, C. A. Mirkin, R. L. Letsinger, Science 289, 1757 (2000).

2. A.A. Lazarides, G. C. Schatz, J. Chem. Phys. 112, 2987 (2000); A.A. Lazarides, G. C. Schatz, J.

Phys. Chem. 104, 460 (2000); J. J. Storhoff, A. A. Lazarides, C. A. Mirkin, R. L. Letsinger, R.

C. Mucic, G. C. Schatz, J. Am. Chem. Soc. 122, 4640 (2000)

. S.-J. Park, A. A. Lazarides, C. A. Mirkin, P. W. Brazis, C. R. Kannewurf, R. L. Letsinger,
Ferroelectric Liquid Crystalline Elastomers:

\section{Novel Materials for Nanomachines}

\section{F. Kremer}

Universität Leipzig, Germany

Ferroelectric Liquid Crystalline Elastomers (FLCE) form a novel class of materials with an extraordinary profile of features: They are ferroelectric and hence piezoelectric, they can be prepared as elastomeric "single crystals" on a macroscopic $(\sim \mathrm{cm})$ scale and they can be obtained as self-supporting thin $(\sim \mu \mathrm{m})$ and (ultra)-thin molecular layers. Furthermore their viscoelastic properties can be tailored by use of a photochemical crosslinking reaction.

The structure and mobility of FCLE is studied by employing polarized, timeresolved Fourier-Transform IR-spectroscopy [1-3]. This enables to analyse in detail the reorientation of the single molecular moieties in the supramolecular system (angle of reorientation, time constant, phase relationship) in response to an external electric field. To measure the (inverse) piezoelectric effect interferometric and $\mathrm{x}$-ray methods are used [4,5]. It is discovered that FLCE in a proper crosslinking density show the highest electrostrictive coefficients which were found up to now for any material. A microscopic interpretation of the observed marcoscopic effects will be presented [5,6].

FLCE offer a strong technological impact especially as materials for sensors and actuators in microsystem technology.

\section{References:}

1. Shilov, S.V.; Gebhard, E.; Skupin,H.; Zentel, R.; Kremer, F.; Macromolecules 32, 1570 (1999) 2. Skupin, H.; Kremer,F.; Shilov,S.V.; Stein, P.;Finkelmann, H.; Macromolecules 32, 3746 (1999) 3. Skupin, H.; Kremer, F.; Shilov, S.; Lehmann, W.; Brodowsky, H.; Gebhard, E.; Zentel, R.; 4. Lehmann, W.; Hartmann, L.; Kremer, F.; Stein, P.; Finkelmann, H.; Kruth, H.; Diele, S.; J. Appl. Phys. 86, (3) 1647 (1999)

5. Kremer, F.; Skupin, H.; Lehmann, W.; Hartmann, L.; Stein, P.; Finkelmann, H.; in press Adv. Chem. Phys. (Ed. J. Vij) (2000)

6. Lehmann, W., Skupin, H., Tolksdorf, C., Gebhard, E., Zentel, R., Krüger, P., Lösche, M. and Kremer, F., Nature, Vol.410, p. 447-450 (2001)

DNA-based positioning of nanoparticles for the realization of a SETdevice

\section{G. Maubach, D. Born, J.M.Köhler, W. Fritzsche}

Molecular Nanotechnology Group, IPHT Jena, Germany

Single-electron tunneling (SET) devices are a promising tool for a future electronic. Their working temperature depends on the size of a metal island. Todays standard lithographic techniques achieve islands in the range of several tens of nanometers, which results in devices only working at low temperatures. For room-temperature devices, islands in the lower nanometer range are needed. One possibility for devices, islands in the lower nanometer range are needed. One possibility for
defined structures of this size are colloidal metal particles. However, their defined structures of this size are colloidal metal
incorporation into microelectronic setups is difficult.

Our project is focused on a method of fine-positioning metal nanoparticles on a micro structured surface using DNA. The microstructures are preferably gold surfaces, which represents nanoelectrodes. Our goal is to stretch a DNA molecule between two electrodes and further use of this molecule to attach a nanobead (or beads) on it, so that the bead can act as metallic island for single-electron tunnelling devices. We are trying different methods for both DNA-attachment and beadpositioning: A DNA with short single-stranded overhangs can be attached via complementary
oligo-nucleotides (which are immobilized on the gold surface) by hybridisation. Anther way is the direct use of thiol-modified DNA, made by implementation of thiol-modified oligonucleotides in a PCR reaction.

The positioning of the nanobead can be realized by triple helix formation. For this purpose we synthesize oligonucleotides, which can form stable triple helix with purpose we synthesize oligonucleotides, which can form stable triple helix with
defined double-stranded DNA molecules. The position of the nanobead is herein defined double-stranded DNA molecules. The position of the nanobead is herein
defined by the sequence of the oligo-nucleotide. Including a branched DNAdefined by the sequence of the oligo-nucleotide. Including a branched DNA-
fragment in the extended DNA molecule, which acts as a template for the binding of fragment in the extended DNA molecule, which acts as a template for the binding of
a nanobead, is a second possibility. The insertion of branched DNA-fragments is a nanobead, is a second possibility.
realized by restriction and ligation.

Gold microstructures for binding and extending of DNA (by use of an electrical field) were prepared and used in DNA-binding experiments. A gap size of $3 \mu \mathrm{m}$ was realized in order to immobilize DNA of about $4 \mu \mathrm{m}$ length (see figure below).
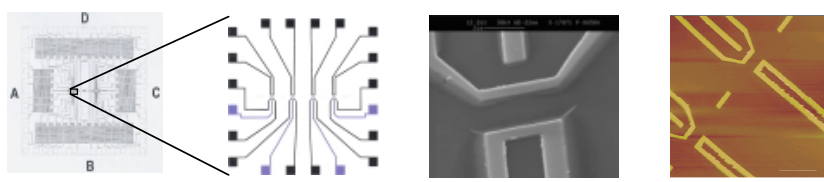
Controlled heterogeneous nucleation of metal clusters on DNA: A novel pathway to fabricate nanowires with quantum behaviour

\section{Michael Mertig, Wolfgang Pompe}

Technical University Dresden, Germany

We report the capability of DNA to control entirely the nucleation of platinum clusters initiated by reduction of a metal salt in solution. The heterogeneous nucleation at the molecule is promoted by the selective chemical activity of DNA bases, to which for example Pt(II) complexes are covalently bound before starting reduction. Theoretical modelling shows that the heterogeneous nucleation is promoted by the strong donor character of the nucleotides, which stabilize newly formed Pt-Pt bonds after a single reduction step. This mechanism allows to suppress completely the competing reaction channel, homogeneous nucleation of metal clusters in solution. As a result, a pure in-place metallization of the DNA template becomes possible, as we demonstrate by the fabrication of ultra-thin cluster chain

Furthermore, we present measurements of the electrical conductivity of metallic nanowires, which have been fabricated by chemical deposition of thin continuous Pd films onto single DNA molecules. The DNA molecules have been positioned between macroscopic Au electrodes and are metallized afterwards. The nanowires exhibit ohmic transport behaviour at room temperature. At low temperature we observe a logarithmic increase of the wire resistance. We assign this behaviour to weak localization of electrons and/or electron-electron interaction processes as it is observed in disordered metallic films.

\section{Scanning Probe Techniques for Single Molecule Studies} Mervyn Miles, Andrew Humphris, Javier Tamayo, Andy Baker, and Jamie
Hobbs

University of Bristol, U.K

The advantages of scanning probe microscopy (SPM) for high-resolution imaging and the ability to image in a variety of environments permitting in situ studies of biomolecular processes are well known. SPM continues to evolve rapidly, not only in these areas, but also in areas not anticipated earlier such as force spectroscopy. In this presentation, recent developments of the application of SPM to biopolymers will be illustrated with examples of atomic-level resolution, and processes of enzymic hydrolysis in biological systems. Important new techniques for biopolymers will be presented including resonance control for the reduction of the probe-specimen force by 100 to 1000 times, a very significant advance in imaging soft or delicate biomolecular structures. Some non-imaging biological applications of the technique will also be discussed.

The minimization of the probe-specimen force is of particular importance in the study of biopolymers and soft materials formed from them such as gels. A recent study of biopolymers and soft materials formed from them such as gels. A recent development know as active Q-control enables the effective quality factor (Q) of the or more. The technique not only results in less damage to delicate specimens, but or more. The technique not only results in less damage to delicate specimens, but
also increases resolution as the structures are deformed less by the probe tip. The also increases resolution as the structures are deformed less
technique also gives strong phase contrast images in liquid.

The mechanics of single molecules under deformation is both dynamic and energetic in nature and so the force measurement contains both conservative (elastic) and dissipative (viscous) components. These two components are associated with different processes within the total molecular deformation process. The capability of separating the measured force into its conservative and dissipative components is of importance in ascribing features in the force-deformation curve to particular molecular events.

A method of measuring this complex quantity for single molecules in a conventional AFM will be presented. The dynamic force sensitivity of the AFM cantilever was increased with an active- $Q$ control, resulting in an effective quality factor of over 300 in a liquid environment. This allowed the tracking of the resonant frequency as 300 in a liquid environment. This allowed the tracking of the resonant frequency as the molecule was stretched and the separation of elastic and dissipative forces. This
method provides an alternative to the transverse dynamic force spectroscopy technique for measuring complex mechanics.

\section{Crystalline Bacterial Cell Surface (S-Layer) Proteins, Secondary Cell Wall Polymers and Lipid Molecules as Building Blocks in Nanobiotechnology}

\section{Dieter Moll, Margit Sára and Uwe B. Sleytr}

Center for Ultrastructure Research/Ludwig Boltzmann-Institute for Molecular Crystalline bacterial cell surface (S-layer) proteins represent the outermost cell envelope component in many bacteria and archaea. S-layers are composed of a single species of protein or glycoprotein subunits, which assemble into lattices with oblique (p1, p2), square (p4) or hexagonal (p3, p6) symmetry. Depending on the lattice type, one morphological unit is composed of one, two, three, four or six subunits. The In the case of bacteria, the S-layer subunits are linked to each other and to the underlying cell envelope layer by non covalent interactions. The S-layer subunits of underlying cell envelope layer by non covalent interactions. The S-layer subunits of anchoring structures in the rigid cell wall layer. Isolated S-layer subunits frequently reassemble in suspension (self-assembly products), or they recrystallise into monolayers on solid supports (silicon wafers, gold, plastic foils), on Langmuir films or on liposomes. Because S-layer proteins are highly anisotropic structures, they can either bind with their outer or inner surface. Functionalisation of S-layer lattices was achieved by covalent binding of enzymes, antibodies or other ligands to the carbodiimide-activated carboxylic acid groups of the S-layer protein. S-layers with immobilized fuation structures and as carrier / adjuvants for conjugated vaccines So far, the genes encoding the S-layer proteins SbsA and SbsB of Bacillus stearothermophilus PV72, SbsC and SbsD of B. stearothermophilus ATCC 12980 and SbpA of Bacillus sphaericus CCM 2177 were sequenced and cloned in Escherichia coli. In the case of SbsC, the structure-function- relationship of distinct segments was investigated in detail by producing various $\mathrm{N}$ - or C-terminally truncated forms. It could be demonstrated that the N-terminal part is responsible for cell wall binding, while the middle part comprises the region required for the selfassembly. In the C-terminal part up to 179 amino acids could be deleted without any
influence on the formation of the regular lattice structure. With SbsB, SbsC and SbpA, the first S-layer fusion proteins were produced, which retained the specific properties of the S-layer protein moieties, as well as those of the fused functional peptide sequence. The first fusion partners included streptavidin, the major birch pollen allergen Bet $\mathrm{v} 1$, and single chain antibodies. Due to the 2D crystalline nature of Slayers, fused functional protein domains or peptide sequences were expected to be presented with a defined spacing and orientation. The streptavidin-SbsB fusion proteins were designed to link the S-layer technology with the streptavidin-biotin technology. Efforts to recrystallise them on silicon and to use them as a template for the self-assembly of arrays of nanoparticles are in progress. The affinity of S-layer proteins to distinct types of secondary cell wall polymers is also exploited for the between pairs of $S$-layer proteins and secondary cell wall polymers were characterised by surface plasmon resonance, and oriented recrystallisation mediated by the secondary cell wall polymer was demonstrated on peptidoglycan containing sacculi. As a next step, solid supports, Langmuir lipid films and liposomes will be functionalised with secondary cell wall polymer, so that they have specific affinity for oriented recrystallisation of S-layer fusion proteins displaying their functionality in

\section{Electrical classification of nanoparticle densities on chip surfaces}

\section{R. Möller, A. Csáki, J. M. Köhler, W. Fritzsche}

Molecular Nanotechnology Group, IPHT Jena, Germany

A scheme for an electrical classification of the solution concentration of bioconjugated colloidal gold particles is presented. It is based on the immobilization of the particles in the gap of microstructured electrodes, followed by a metal enhancement step and electrical measurements. The surface density of particles depends on the solution concentrations, and the metal enhancement classifies this density by yielding conductive surfaces only for densities above a threshold. The growth of the nanoparticles was characterized using scanning electron microscopy and scanning force microscopy. Size enhancement ratios of up to 10 were observed for $30 \mathrm{~nm}$ particles, and could be controlled by the incubation time.

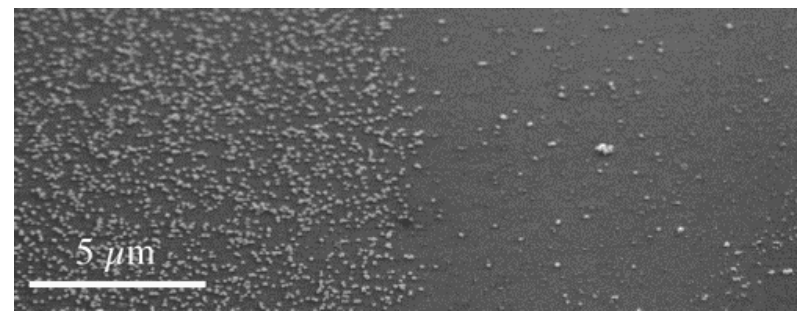

Scanning electron micrograph of a substrate with metal-enhanced (left) and unmodified (right) $30 \mathrm{~nm}$ gold nanoparticles. 


\section{Semisynthetic DNA-Protein Conjugates:}

\section{Applications in Life-Sciences and NanoBiotechnology}

\section{Christof M. Niemeyer}

\section{Universität Bremen, FB 2 - UFT, Germany}

The biomimetic bottom-up assembly of programmed molecular building blocks provides a novel strategy for the generation of nanomaterials. With respect to these efforts, DNA is a highly promising material for the fabrication of nanostructured supramolecular architecture [1]. We have carried out extensive scanning force microscopy studies on a novel class of semisynthetic DNA-protein conjugates, self-assembled oligomeric networks consisting of streptavidin and double-stranded DNA [2]. These nanostructured assemblies are applicable as high performance reagents in immuno-PCR, a very sensitive technique for the trace analysis of proteins and other antigens. This allows, for instance, the detection of the immunomodulating and antitumor drug concentrations not detectable by conventional ELISA assays. Moreover, the oligomeric DNA-streptavidin networks can be converted to well-defined supramolecular nanocircles [3], applicable as modular building blocks for the generation of novel immunological reagents [4], the construction of nanometerscaled "soft material" standards for scanning probe microscopy [5], and other fields of nanobiotechnology.

Another example concerns covalent conjugates of single-stranded DNA oligomers and streptavidin [6], which can be utilized as biomolecular adapters for the immobilization of biotinylated macromolecules on surfaces via nucleic acid hybridization. This technique, DNA-directed immobilization (DDI), not only proceeds with greater immobilization efficiency than conventional adsorption methods, it also allows for reversible and site-selective functionalization of solid supports with enzymes, immunoglobulins and other compounds. Thus, DDI is particularly suitable for the fabrication of microstructured, reusable protein biochips and other sensor devices [7]. In addition, the covalent DNA-streptavidin conjugates are also convenient for constructions on the nanometer-scale since they allow for selective positioning of biotin-derivatized molecules along a singlestranded nucleic acid carrier molecule. For instance, gold nanoparticles and antibodies can be assembled to form well-defined functional biometallic nanostructures usefull, for instance, as diagnostic tools in biosensor applications [8]. [1] Niemeyer Curr. Opinion Chem. Biol. 2000, 4, 609; [2] Niemeyer et al. Nucleic Acids Res. 1999, 27, 4553; [3] Niemeyer et al. Angew. Chem. Int. Ed. 2000, 39, 3055; [4] Niemeyer et al.
Angew. Chem. Int. Ed. 2001, 40, in press; [5] Song et al. ChemPhysChem 2001, 2, in press; [6] Angew. Chem. Int. Ed. 2001, 40, in press; [5] Song et al. ChemPhysChem 2001, 2, in press; [6] 268, 54; [8] Niemeyer et al. Angew. Chem. Int. Ed. 1998, 37, 2265.

\section{Entrapping Biological Systems in Polyelectrolyte Nanostructured Capsules}

D. Silvano, O. Cavalleri, A. Diaspro, S.Krol, A. Gliozzi

INFM and Department of Physics, University of Genoa, Italy.

Oppositely charged polyelectrolytes can be adsorbed onto nano- and micrometer sized particles. By using degradable cores, one can obtain hollow polyelectrolyte shells, also known as nanocapsules $[1,2]$.

These shells are of both biological and medical interest, since they are very stable and with tunable transport properties, due to their controllable nanostructured interface. For instance, it is possible to modulate the shell permeability by interface. For instance, it is possible to modulate the shell permeability by
changing the $\mathrm{pH}$ or by using different kind of cores. Such model systems can be changing the $\mathrm{pH}$ or by using different kind of cores. Such model systems can be entrance of substances. The same technique allows also to functionalize the capsule surface. One can envisage many applications for biofunctionalized shells ranging from targeted drug release to the study of interactions between biomolecules.

Another interesting aspect is the use of this technique to coat living cells. We coated Saccharomyces cerevisiae cells with polyelectrolyte multilayers. The goal is to create a protected microenvironment for still alive cells.

Hollow capsules and coated cells have been prepared by alternative adsorption of poly-(styrenesulfonate, sodium salt) (PSS) and poly-(allylamine hydrochloride) (PAH). The adsorption of proteins on charged shells has been investigated. The structure and the permeability properties of shells biofunctionalized by protein coating have been examined by two-photon excitation [3] and confocal coating have been examined by two-photon excitation [3] and confocal microscopy while keeping the shells in the hydrated state. This also allows to follow the process of core dissolution and to utilize two-photon excitation in an active way for partial or total core removal, for photochemistry in confined volume and to study influence of spatial organization of receptors. The morphology of the shells and the texture of their walls before and after the protein adsorption has been characterized by scanning force microscopy measurements on dried capsules adsorbed on mica. With the confocal techniques, we also tested the yeast cells viability during and after the coating process.
Nanostructures for the characterization of nanoclusters \& molecules

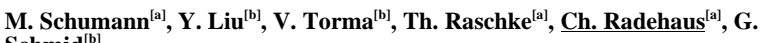
Schmid $^{[b]}$

${ }^{[a]}$ Technische Universität Chemnitz, Germany

${ }^{[b]}$ Institut für Anorganische Chemie, Universität Essen, Germany

Because of the continuous downscaling of CMOS - devices, memories etc. and the increasing problems with key processes, such as lithography processes or the increasing problens wh measurent those based on nanoclusters or molecules become nore and more important and could at leas $\mathrm{nm}$. The examination of the electrical behavior of one or more single clusters or $\mathrm{nm}$. The examination of the electrical
molecules requires nanostructures.

In our approach we present nanostructures with a minimum feature size below 50 $\mathrm{nm}$ and a contact gap between $10-50 \mathrm{~nm}$. These structures consist of tungsten electrodes, etched from a $25 \mathrm{~nm}$ thick tungsten layer on a silicon wafer with a 80 $300 \mathrm{~nm}$ thick thermal oxide using an electron beam lithography process based on a negative resist and a $\mathrm{SF}_{6}-\mathrm{RIE}$ etch technology. We demonstrate the electrical properties of sputtered Ag-clusters as well as $\mathrm{Au}_{55}\left(\mathrm{PPh}_{3}\right)_{12} \mathrm{Cl}_{6}$-clusters showing single electron tunneling behavior at room temperature as well as the diode behavior of $\mathrm{Au}_{55}$ - cluster monolayers.

\section{Physical Properties Determining Self-Organization of Motors and Microtubules}

\section{Thomas Surrey, François Nédélec, Stanislas Leibler, Eric Karsenti}

European Molecular Biology Laboratory, Heidelberg

A remarkable property of the cytoskeleton of living cells resides in the versatility of patterns it can produce. Similar sets of proteins become organized into very different assemblies depending on the cell type and on the cell cycle stage. Filaments and mechanochemical enzymes (motor proteins) play a crucial role in determining the intracellular architecture. Here, we analyze the collective contribution of multiple parameters to the definition of patterns generated by microtubule-filaments and oligomeric motors. We study experimentally which patterns can be generated in vitro by mixtures of purified proteins. We find that mixtures with a single motor species can create microtubule asters and vortices while mixtures containing two motors of opposite directionality can generate interconnected networks. In a theoretical study, we reproduce the experimental results numerically and explore the parameter space of pattern formation by computer simulations. Our results emphasize the structural role of kinetic parameters in self-organizing motor/ microtubule systems.

Ref.: Science 292, 1167-1171 (2001)

1. E.Donath, G.B.Sukhorukov, F.Caruso, S.A.Davis, H.Mohwald. Angew.Chem.Int.Ed., 16, 2202-2205 (1998).

. S.Leporatti, A Voigt, R.Mitlohner, G.Sukhorukov, E.Donath, H.Mohwald. Langmuir, 16, 4059-4063 (2000)

3. A.Diaspro, M.Robello. J.Photochem.Photobiol. B, 55, 1-8, (2000). 


\section{Development of biomolecular nano-step shuttles}

\section{Eberhard Unger, Konrad J. Böhm}

Institute for Molecular Biotechnology Jena, Germany

Eukaryotic organisms express several types of motor proteins to realize diverse intracellular transport and motility processes. These proteins, among them the kinesin, conert the ching al eng mis on kinesins transport specialized cargoes ( $e$. neuronal vesicles, chromosomes) to their cellular destination. The direction of transport depends on both microtubule polarity and the intrinsic molecular kinesin structure.

Purified kinesin is able to generate force also outside cells. The kinesin-based

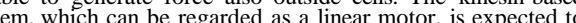
contribute to the development of novel nanoactuatoric devices working with nanometre-precise feed rates. Such a device might be a molecular shuttle which conveys specific particles as cargoes to predetermined points. Practical usage predicts the solution of some methodological and technical problems, including velocity regulation, temporal stability, fuel supply, force generation into a desirec direction, on and off switching, the determination of suitable materials, of the tolerable surfaces roughness, and of the minimal free vertical space still enabling The present paper reviews our recent results that contribute to find a basic configuration for constructing a kinesin-driven shuttle device: Using gliding microtubules as a model, we demonstrated that the transport velocity increased trom about $0.6 \mu \mathrm{m} / \mathrm{s}$ to $2.0 \mu \mathrm{m} / \mathrm{s}$ by raising molar $\mathrm{Mg}^{2}$ ATP ratio, elevating temperature, or lowering kinesin surface density [1]. In contrast, polyhydroxy space of about $100 \mathrm{~nm}$ height [3] and works with constant velocity for hours under conditions of sufficient energy (ATP) supply. Individual gliding microtubules overcame surface height differences up to about $280 \mathrm{~nm}$. Kinesin was observed to generate motility on a variety of technically relevant materials, including glass, quartz, silicon, carbon, gold, and polystyrene.

Gliding microtubules can be aligned in isopolar fashion applying flow fields [3] After alignment the microtubules were chemically fixed [4], resulting in stable arrays of microtubule rails suitable for the unidirectional transport of kinesincoated cargoes with dimension up to $20 \mu \mathrm{m}$. The cargo (even a small one, e.g. 100-nm beads) can change from one microtubule to an adjacent one, enabling transport distances in millimetre ranges, Significantly exceeding the length of the theoretically non-limited length might be assembled, which can be used for the
directed transport of different cargoes. To establish a shuttle system, parallel threads of microtubule rails with opposite polarity have to be immobilized onto the substrate.

Böhm K.J., Stracke R., Unger E. (2000) Cell Biol. Int. 24 335-341

Böhm K.J., Stracke R., Vater W., Unger E. (2001) in Micro- and Nanostructures of Biological Systems (eds. Hein H.-J., Bischoff G.) Shaker Verlag Aachen, pp. 153-165
Stracke R., Böhm K.., Burgold J., Schacht H J., Unger E (2000) Nanotechnol. 11 52-56

[4] Turner D., Chang C.Y., Fang K., Cuomo P. Murphy D. (1996) Anal. Biochem. 242 20-25

Orientation of Quadruplex DNA (G-wires) and purported G-wire crystals on Mica.

Bethany Rioux, Matthew Fletcher and James Vesenka

University of New England, Biddeford (ME) U.S.A.

G-wires are four stranded DNA polymers formed by the self-assembly of simple, G-rich oligomersi. G-wires incubated in a growth medium and adsorbed onto mica appear to orient themselves relative to the mica surface structure. Atomic
force microscopy and low current scanning tunneling microscopy indicate that Gwires become oriented parallel to the next nearest neighbor sites on mica surfaces.. Control experiments with duplex DNA of similar length to the G-wires show no orientation preference. A proposed model, based on the lattice match nearest neighbor sites of mica $(0.90 \mathrm{~nm})$, is used to explain the preferential orientations (Fig. 1)

A growth kinetics study was used to establish a timeline for the assembly of sufficiently long G-wires for study as possible electrically conducting "nanowires". The robust and flexible character of duplex DNA has long been examined for its potential to address the molecular wire question. Very low conductivity through short DNA sequences $(\sim 15$ base pairs $)$ has been established by photo-induced and flash-quench techniques ${ }^{11}$ while electron hopping has been established as the dominant form of electron migration in longer segments of DNA". Under hydrated conditions, LCSTM reveals that duplex DNA is a poor DNA appears to collapse on the surface of mica and other silicates (e $\mathrm{g}$ glassware)". Such behavior would affect electron transfer along the twisted glassware) Suckone-shape ${ }^{v i}$, raising continuity concerns. Biomolecular templates have been employed as masks and scaffolding to create traditional miniature metalized conductors. However, the large grain-size of the evaporated metals make the resulting structure irregular, and thus commercially problematic $c^{\text {vi. }}$. An ideal "nano-wire" would combine the flexibility of a biopolymer, the uniformity of integrated circuit technology and reproducible conductivity of metals. The caged metal cations integrated into a hydrated G-wire molecule may have some (estimated between 0.3-0.7 met to cations are sufficiently close to each other -bonding of adjacent Guanine-quartets may overlap enough to enhance electron mobility.

After about a month of growth the G-wire cocktail yielded structures that oriented themselves along the exact same directions as the G-wires. These long, straight structures appear to be crystalline in nature (Fig. 2). The height of a single layer of these crystals is a little more than half of the non-crystalline Gwires $(1.4 \mathrm{~nm}$ compared to $2.2 \mathrm{~nm}$ ). However, when two layers of the crystals appear they are NOT double in height, but exactly $2.2 \mathrm{~nm}$ ! We speculate that these structures may be some form of two-dimensional G-wire crystals. studies (micrometer lengths).

'Marsh, T.C., J. Vesenka, and E. Henderson. 1995. A new DNA nanostructure, the G-wire, imaged by scanning probe microscopy.

Nucleic Acids Res. 23: $3696-700$.
${ }^{1}$ T.J. Meade \& J.F. Kayyem, Angew. Chem. Int. Engl. 34, 352 (1995).

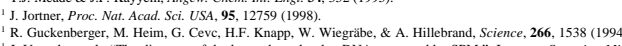

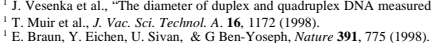

Nanolithography processing of thin stable polymer films for molecular technology

V.A. Bykov (a), A. A. Valyaev (a), H. Lee (b).

(a) State Research Institute of Physical Problems Moscow, Zelenograd, Russia (b) Hanyang University, Seoul, South Korea

SPM (Scanning Probe Microscopy) local oxidation on semiconductor and conductive surfaces by anodization technique is a promising nanolithography approach for the fabrication of nanometer-scale electronic devices and was presented in several works earlier. The formation of an accurate image on polymer substrate is quite modern and the results can be applied for a creation of molecular devices. This method was realized on single-layer stable organic films on silicon substrate by using of P-47H device of NT-MDT and silicon cantilevers with TiN conductive coating. The main feature of the experiment is in the regimes of formation of the picture. Usually contact mode is used for a nanolithography operations, but this method drastically degrade the conductive coating on the cantilevers and mechanical pushing of some polymer films is possible during cantilevers and mechanical pushing of some polymer films is possible during oxidation in contact with the probe. Semicontact mode operation allows us to
make more accurate image in comparison with the contact mode and the same probe was successfully used in order to form many of such pictures.

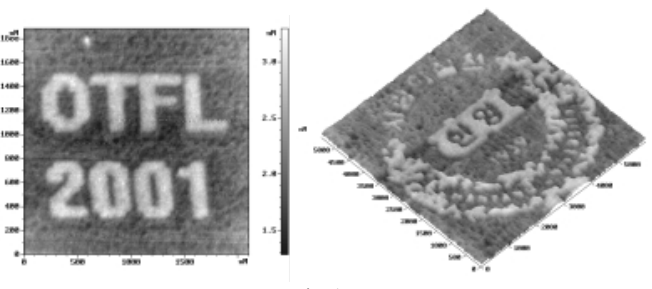

Fig 1

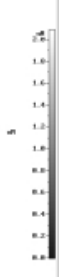

Fig 2.
Electrical lithography on mixed LB films of HDA-PA on silicon wafer. Applied pulse voltage- 10 volts, pulse duration-12-30 ms.

Spin-coated organic films, Langmuir-Blodgett films and self-assembled monolayers were modificated by electron beam induced from the tip of AFM. The resolution of this technique is greatly depended on (1) the value of applied voltage (2) scanning rate (3) humidity (4) substrate and probe material properties. The results of this investigations are presented and discussed.
Semiconductor nanocrystals for nanoelectronics - studied by STM, SEM and AFM

K. Walzer (1), U. Quaade (1), K. Stokbro (1), N.C. Greenham (2), D.S.
Ginger (2)

(1) Mikroelektronik Centret, Technical University of Denmark, DK-2800 Lyngby (2) Cavendish Laboratory, Cambridge CB3 OHE, United Kingdom

The top-down approach in structuring electronic devices becomes more and more difficult when approaching the $10 \mathrm{~nm}$ scale. Also most of the bottom-up strategies discussed at the moment are still not feasible. A way out could be use of a combined technique, which uses conventionally structured semiconductor devices as a basis, where additional smaller structures are self-assembled. Nanocrystals are promising systems for such an application in molecular size charge storage promising
devices.

Using scanning tunneling microscopy and spectroscopy, we study the distribution and the local electronic properties of these substances, adsorbed onto industrially relevant substrates, like silicon and gold. We use monodisperse CdSe nanocrystals, $3.1 \mathrm{~nm}$ in diameter, covered with an organic passivation shell of TOPO (= trioctyl-phosphine-oxide), which prevents the nanocrystals from sticking together and isolates them electrically. Distribution experiments carried out on hydrogen passivated $\mathrm{Si}(100)$ show that the nanocrystals applied in submonolayers do not spread homogeneously over the surface. Instead, they form small snowflake shaped arrangements of 2 to $5 \mu \mathrm{m}$ in diameter and 3 to $10 \mathrm{~nm}$ in height. Individual nanocrystals can be observed by STM. As expected, tunnel spectra collected on such nanocrystals show a widening of the band gap with respect to the bare substrate. However, also a pinning of the particle's HOMO to the valence band of the substrate is observed. We discuss a possible explanation for this behaviour. 
Resonance Light Scattering Particles as Fluorescent Analog Labels for Ultrasensitive Analyte Detection

\section{Juan Yguerabide and Evangelina E. Yguerabide}

Genicon Sciences Corporation, San Diego, USA

When a $40 \mathrm{~nm}$ gold particle suspension is illuminated by a white beam of light, the scattered light looks like fluorescence. It has a clear, green color. These, as well as other observations, have suggested to us that resonance light scattering (RLS) particles can be used as fluorescent analog labels for analyte detection in biochemistry, cell biology, molecular biology and laboratory clinical diagnostics. RLS particles such as gold and silver particles are advantageous because they have very high light scattering powers. A $60 \mathrm{~nm}$ gold particle has a light producing power equivalent to abo particles do not photobleach. $60 \mathrm{~nm}$ particles can be detected by light scattering Detection can by the unaided eye (qualitative) or simple photodetectors (quantitative) . Individual particles can be detected visually in a student's microscope using simple dark field illumination which allows for single particle detection. RLS particle labels thus allow ultrasensitive detection using very simple methods of illumination and detection Moreover, particles that scatter different colors of light can be obtained by changing particle size or composition which allows for multiplexed detection of several analytes in a mixture. Antibodies, DNA probes and other detection substances can be readily attached to the RLS particles for selective detection of specific analytes. We have successfully applied our RLS particle technology to a wide range of assays including solid phase immuno- and DNA probe assays (including microarrays) homogeneous liquid phase assays, detection of surface receptors in cells and tissues and in situ hybridization.
DNA supramolecular complex - natural lipid-bound DNA as studied by single molecule imaging approach

R. Zhdanov ${ }^{1}$, G. Bischoff ${ }^{2}$, R. Bischoff ${ }^{3}$, N. Strazhevskaya ${ }^{1}$, A. Shmyrina ${ }^{1}$, A. Elkady ${ }^{1}$, A. Moskovtsev ${ }^{1}$ and V. Struchkov

${ }^{\prime}$ V.N. Orekhovich Institute of Biomedical Chemistry, Russian Academy of Medical Sciences, 10, Pogodinskaya St., Moscow 119992 Russian Federation, Institute of Biochemistry, Martin-Luther University Halle/Saale, Germany,

SENSOBI Sensoren Gmbh, Halle/Saale, Germany

${ }^{4}$ N.N. Blokhin National Oncological Center, 24, Kashirskoe shaussee, Moscow 115478 Russian Federation

Neutral and phospholipids represent the important components of chromosomes and DNA. The natural lipid-bound DNA - supramolecular DNA complexes (SM-DNA) were isolated at the first time from rat thymus and liver in 1974. This type of complexes was isolated then from other mammalian cells, tumor cells, bacteria and phages. The area of research of natural DNA-lipid (membrane) complexes became present very acute because of importance of general principles of their structure and fresent ve to dion new non-viral gene delivery systems for gene the sture an The The natura De involved nto regulation of gene expression along with proteins and methylation of nucleic acid lipid-DNA genomic code. lipid-DNA genomic code.

t was demonstrated that lipids in SC-DNA are presented as two pools: the pool of lipids loosely bound to the DNA $(\sim 60 \%)$, and the pool of tightly bound to the DNA $(40 \%)$. The loosely bound lipids are extracted from the SC-DNA under mild reatment: with $35 \%$ aqueous solution $\left(24 \mathrm{hrs}, 37^{\circ} \mathrm{C}\right.$ without stirring). In opposite, the tightly bound lipids can be extracted with chloroform/methanol (2:1) mixture only after incubation of residual DNA with DNase $\mathrm{I}\left(1 \mathrm{hr}, 37^{\circ} \mathrm{C}\right)$. It was found that the DNA bound lipids of eukaryotic and prokaryotic cells contain neutral lipids and phospholipids. Phospholipids from eukaryotes consist of at least 5 individual fractions: cardiolipin, CL (40-50\% of total phospholipids), phosphatidylethanolamine, PE (25$30 \%)$, phosphatidylcholine, PC (15-20\%), phosphatidylserine, PS (5-7\%), phosphatidylinositol, PI (3\%), and only traces of sphingomyelin, SM, or phosphatidic acid, PA. In opposite, DNA-bound phospholipids from E.coli B and phage T2 are only presented by CL $(60-70 \%)$ and PE $(30-40 \%)$. Neutral lipids from normal eukaryotic cells, Ecoli B and phage T2 contain four individul fractions: fatty acid esters of ty acid esters of holesterol, CholE (38-40\%), free fatty acids, FFA (20-25\%), diglycerides, DG (25, and free cholesterol, Chol $(8-10 \%)$. Thus, the DNA-bound lipids have an unusual specific composition which differs essentially from those of nuclear membrane, chromatin, and nuclear matrix lipids, where the major lipids are presented by cholesterol, PC and SM.

Scanning atomic force and tunnel microscopies are applied to study the structure of SM-DNA and to recognize 4 DNA double helixes inside SM-DNA, and lipid molecules - inside DNA double helix. These techniques are considered as perspective tools for step-by-step lipid sequence decoding of lipid-bound DNA.
Immobilization of PCR amplified HBV core gene and hybridization detection for genochips

\section{Guo-Jun Zhang, Dai-Wen Pang}

Department of Chemistry, Wuhan University, Wuhan, 430072, P.R.China

The rapid detection of specific DNA sequences is of great importance for a variety of biomedical and biotechnological applications. Genochips technology developed recently has been successfully applied to the simultaneous expression of many thousands of genes and to large-scale gene discovery, as well as polymorphism screening and mapping of genomic DNA clones ${ }^{[1]}$. Development of genochips relies heavily on the effective immobilization of DNA
onto glass surfaces. Many methods were reported for covalent attachment of onto glass surfaces. Many methods were reported for covalent attachment of
oligonucleotides ${ }^{[2-3]}$. However, the immobilization of genome DNA and big oligonucleotides
fragment DNA is also important in clinical applications.

We report here the immobilization of polymerase chain reaction (PCR) amplified Hepatitis B virus (HBV) core gene and hybridization detection for genochips. C gene fragments in HBV genome were chosen as specific fragments. The primers were modified with amino group and FITC at the $5^{\prime}$ end, respectively. DNA samples were extracted from serum from clinical individuals, and PCR was used to amplify the DNA probes and complementary target molecules modified with amino group and FITC at the $5^{\prime}$ end, respectively. The DNA probes modified with amino group at the $5^{\prime}$ end were covalently immobilized onto the surface via

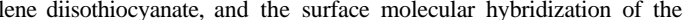
immobilized probes with complementary target molecules modified with FITC at the $5^{\prime}$ end was monitored with an inverted fluorescence microscope (Model IX70) coupled with a cooled-CCD. The hybridization showed that 3 patients positive for HBV surface antigen had positive results under fluorescence microscope. The investigated system has several advantages including specificity

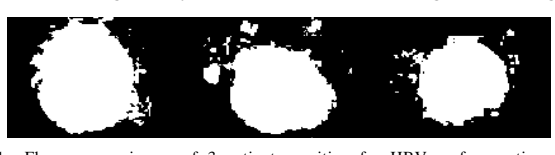

Fig. 1 Fluorescence image of 3 patients positive for HBV surf
via hybridization for detecting HBV DNA in the serum by PCR

for hybridization, fast detection and may meet the requirements of clinical diagnosis.

References:

Ramsay, G. (1998) Nature Biotech 16, 40-44

22(24), 5456-5465.

Zhang, G. J., Pang, D. W, Liu, B. F., Lv, Y. T. (2001) Acta Chimica Sinica in press. 